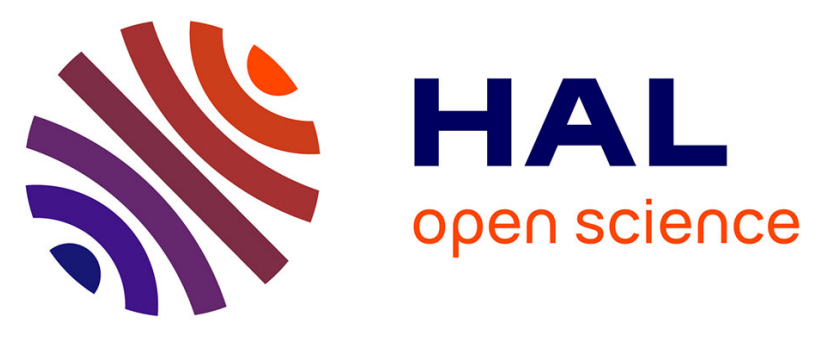

\title{
Contribution de la mesure de la pression intra-orale pour la compréhension des troubles de la coordination pneumophonique dans la dysarthrie parkinsonienne
}

Moustapha Sarr, S. Pinto, Ludovic Jankowski, B. Teston, Alain Purson, Alain Ghio, Jean Regis, Jean-Claude Peragut, François Viallet

\section{To cite this version:}

Moustapha Sarr, S. Pinto, Ludovic Jankowski, B. Teston, Alain Purson, et al.. Contribution de la mesure de la pression intra-orale pour la compréhension des troubles de la coordination pneumophonique dans la dysarthrie parkinsonienne. Revue Neurologique, 2009, 165 (12), pp.1055 - 1061. 10.1016/j.neurol.2009.03.012 . hal-01616004

\section{HAL Id: hal-01616004 https://hal.science/hal-01616004}

Submitted on 19 Apr 2018

HAL is a multi-disciplinary open access archive for the deposit and dissemination of scientific research documents, whether they are published or not. The documents may come from teaching and research institutions in France or abroad, or from public or private research centers.
L'archive ouverte pluridisciplinaire HAL, est destinée au dépôt et à la diffusion de documents scientifiques de niveau recherche, publiés ou non, émanant des établissements d'enseignement et de recherche français ou étrangers, des laboratoires publics ou privés. 
Provided for non-commercial research and education use. Not for reproduction, distribution or commercial use.

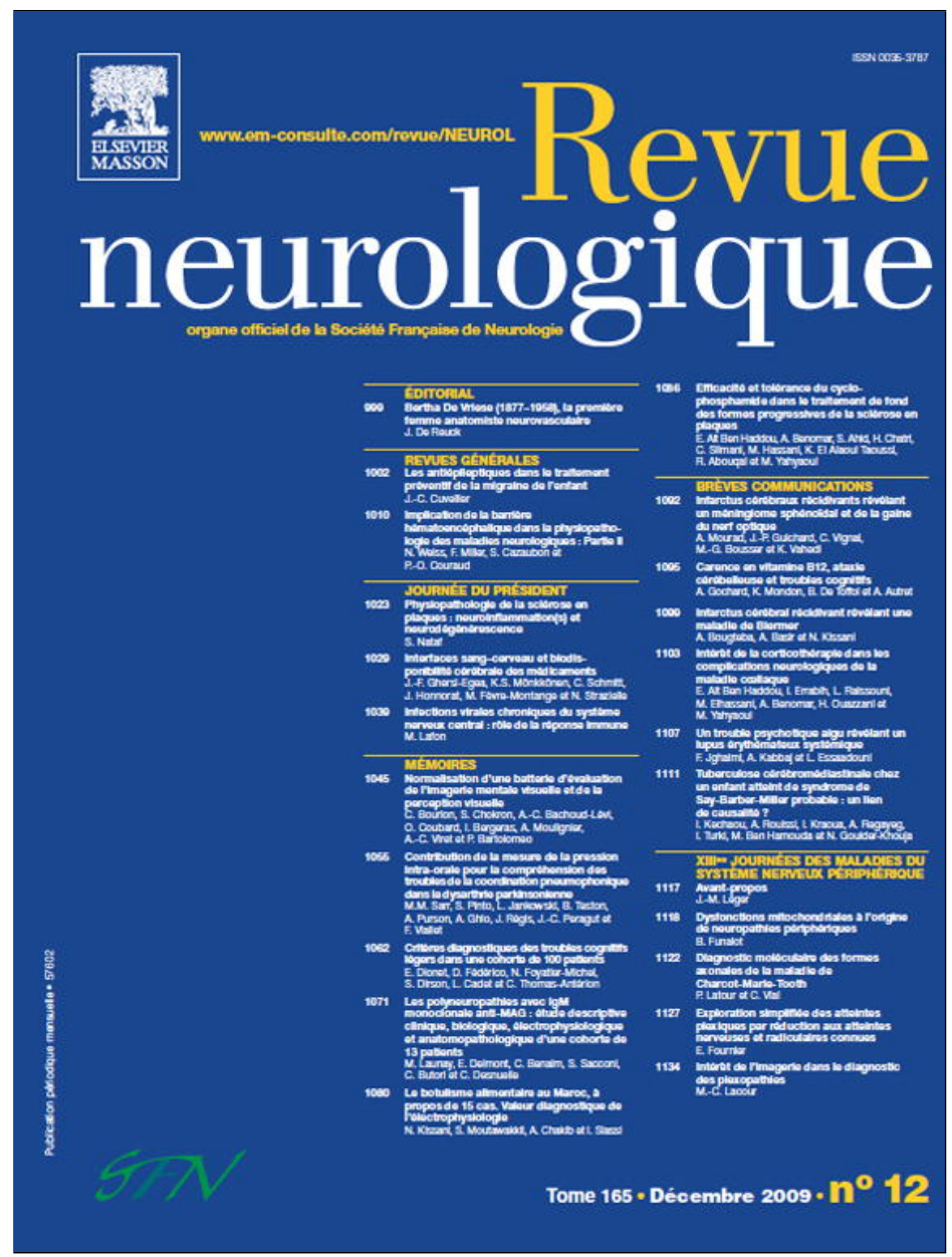

This article appeared in a journal published by Elsevier. The attached copy is furnished to the author for internal non-commercial research and education use, including for instruction at the authors institution and sharing with colleagues.

Other uses, including reproduction and distribution, or selling or licensing copies, or posting to personal, institutional or third party websites are prohibited.

In most cases authors are permitted to post their version of the article (e.g. in Word or Tex form) to their personal website or institutional repository. Authors requiring further information regarding Elsevier's archiving and manuscript policies are encouraged to visit:

http://www.elsevier.com/copyright 
Disponible en ligne sur

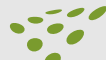

www.sciencedirect.com
Elsevier Masson France

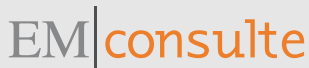

www.em-consulte.com

Mémoire

\title{
Contribution de la mesure de la pression intra-orale pour la compréhension des troubles de la coordination pneumophonique dans la dysarthrie parkinsonienne
}

\section{Measurement of intra-oral pressure as a contributor for the understanding of pneumophonic coordination impairment in Parkinsonian dysarthria}

\author{
M.M. Sarr ${ }^{a, *, b}$, S. Pinto ${ }^{b}$, L. Jankowski ${ }^{b, c}$, B. Teston ${ }^{b}$, A. Purson $^{b, c}$, A. Ghio $^{b}$, J. Régis ${ }^{d}$, \\ J.-C. Peragut ${ }^{d}$, F. Viallet ${ }^{b, c}$ \\ ${ }^{a}$ Laboratoire de physiologie médicale et d'explorations fonctionnelles, faculté de médecine, université Cheikh Anta Diop, Dakar, Sénégal \\ ${ }^{\mathrm{b}}$ UMR 6057 CNRS, laboratoire parole et langage, université Aix-Marseille, Aix-en-Provence, France \\ ${ }^{\mathrm{c}}$ Service de neurologie, CHR du Pays-d'Aix, Aix-en-Provence, France \\ ${ }^{\mathrm{d}}$ Service de neurochirurgie stéréotaxique et fonctionnelle, hôpital Timone, Marseille, France
}

I N F O A R T I C L E

\section{Historique de l'article :}

Reçu le 23 juillet 2008

Reçu sous la forme révisée le

10 octobre 2008

Accepté le 24 mars 2009

Disponible sur Internet le

29 avril 2009

\section{Mots clés}

Maladie de Parkinson

\section{Dysarthrie}

Coordination pneumophonique

Pression intra-orale

Stimulation du noyau

subthalamique

\section{Keywords:}

Parkinson's disease

Dysarthria

Pneumophonic coordination

Intraoral pressure

Subthalamic nucleus stimulation

\section{R É S U M É}

Introduction. - La stimulation du noyau subthalamique (NST) dans la maladie de Parkinson (MP) constitue un espoir thérapeutique mais elle ne semble pas franchement améliorer les signes axiaux dont la dysarthrie. L'objectif de ce travail a été d'évaluer les effets de la stimulation du NST sur le décours de la pression intra-orale (PIO) pendant la phase expiratoire.

Patients et méthode. - Vingt patients parkinsoniens ont été enregistrés en conditions ON et OFF stimulation pour évaluer la PIO sur trois points de mesure pendant la réalisation d'une phrase correspondant à un groupe de souffle. Onze sujets contrôles (CTRL) ont aussi été enregistrés.

Résultats. - La stimulation du NST améliorait significativement la PIO au niveau des deux premiers points de mesure mais pas au niveau du troisième point où un effet de convergence était observé. De surcroît, la performance des patients ON STIM restait très en deçà de celle des sujets CTRL pour les trois points de mesure.

Conclusion. - La mesure de PIO peut être considérée comme un indicateur pertinent pour l'évaluation des troubles de la parole liés à la MP. Elle n'est améliorée par la stimulation du NST que dans la partie initiale du décours de la phase expiratoire, confirmant l'effet modeste de la stimulation du NST sur les troubles axiaux.

(C) 2009 Elsevier Masson SAS. Tous droits réservés.

\footnotetext{
* Auteur correspondant.

Adresse e-mail : taphasarr@gmail.com (M.M. Sarr).

0035-3787/\$ - see front matter (C) 2009 Elsevier Masson SAS. Tous droits réservés.

doi:10.1016/j.neurol.2009.03.012
} 


\section{S U M M A R Y}

Introduction. - Parkinsonian dysarthria can alter oral communication of the patients in the long-term. Subthalamic nucleus (STN) stimulation represents an interesting therapeutic option, although it does not seem to improve axial signs, of which dysarthric speech. The objective of our study was to contribute to the evaluation of STN stimulation effects on speech impairment and in particular on pneumophonic coordination: this latter parameter can be assessed indirectly by evaluating the temporal progression of the intraoral pressure (IOP) during the expiratory phase; thus, IOP represents the transient expression of subglottal pressure (SGP).

Patients and method. - Using a dedicated system (EVA2), 20 parkinsonian patients were recorded in ON and OFF STN stimulation conditions in order to evaluate IOP on three measurement points (2nd, 4th and 6th consonants $\mathrm{P}$ ) during realization of the sentence "Papa ne m'a pas parlé de beau-papa" ("Daddy did not speak to me about daddy-in-law") which corresponds to a breath group. Eleven control subjects were recorded in parallel in order to define reference measurements.

Results. - STN stimulation improved significantly IOP at the level of the initial measurement points (2nd P and 4th P), with an effect of convergence at the level of the third point (6th P) where the difference between OFF and ON STIM conditions was not significant any more. In addition, the performance of the patients ON STIM remained much lower than that of the control subjects.

Conclusion. - Our results raise the significant concept that IOP measurement can be regarded as a relevant indicator for dysarthria in Parkinson's disease. They also show that the improvement of pneumophonic coordination by STN stimulation is restricted to the initial period of the expiratory phase, confirming again the mitigated and controversial effect of STN stimulation on axial signs.

(C) 2009 Elsevier Masson SAS. All rights reserved.

\section{Introduction}

La maladie de Parkinson idiopathique (MPI) est classiquement caractérisée par une triade symptomatique pouvant associer tremblement de repos, rigidité et bradykinésie (Vercueil, 2000). Toutefois, au cours de son évolution naturelle, qui se fait inexorablement vers le déclin moteur (Viallet et al., 2001), il s'y greffe un certain nombre de troubles axiaux parmi lesquels ceux liés à la parole retiennent particulièrement notre attention. Ces derniers, regroupés sous le vocable descriptif de " dysarthrie hypokinétique » (Darley et al., 1969), doivent susciter un intérêt certain dans la mesure où ils représentent un facteur majeur de handicap hypothéquant à long terme la communication orale du patient. Le qualificatif hypokinétique fait à la fois référence à la réduction des mouvements articulatoires et à la diminution de la modulation prosodique. D'ailleurs, l'histoire naturelle de cette dysarthrie a été bien précisée en ces termes :

« les troubles de la parole deviennent de plus en plus évidents (...) Les points d'accentuation disparaissent, le volume vocal diminue, tandis que la prononciation des consonnes se dégrade et que la phrase se termine dans un murmure " (Selby, 1968).

La voix faible et monotone au début de la maladie devient, avec l'aggravation progressive, inaudible et inintelligible. Ces troubles relèvent, comme les autres signes, du déficit en dopamine responsable d'une incompétence phonatoire par hypokinésie des muscles laryngés et respiratoires avec une réduction de la pression sous-glottique (PSG) (Viallet et Teston, 2007). Lors de la production de parole, un mouvement d'air dans le conduit vocal vient exercer une pression sur les cordes vocales qui ont tendance à se fermer et donc à s'opposer au passage de l'air. La pression exercée par l'air expiratoire sur les cordes vocales n'est rien d'autre que la PSG ; quand elle devient supérieure à la résistance laryngée, les cordes vocales s'ouvrent et laissent passer l'air. Ce passage d'air entraîne une diminution de la PSG ; dès lors, les cordes se referment sous l'effet de leur élasticité propre. Une fois les cordes vocales fermées, le processus cyclique peut recommencer. Donc, la génération du son résulte d'un phénomène périodique (vibration laryngée) lui-même issu de la transformation d'une pression continue exercée par la colonne d'air expiratoire sur les cordes vocales (PSG) (Crevier-Buchman, 2007 ; Solomon, 2007). La production de parole est basée sur cette coordination pneumophonique complexe, reposant elle-même sur une relation étroite entre la vibration laryngée et la PSG.

Notre objectif était, d'une part, d'étudier l'évolution de la PSG dans la dysarthrie parkinsonienne afin de tester l'hypothèse qu'une chute importante de la PSG pendant son décours dans le cycle expiratoire serait un indice robuste de dysarthrie. D'autre part, l'effet de la stimulation du noyau subthalamique (NST) sur ce paramètre important de la coordination pneumophonique a également été considéré. En effet, la stimulation du NST est un modèle d'investigation intéressant en ce sens qu'elle cible des patients avec peu ou pas de déclin cognitif, donc avec une dénervation dopaminergique relativement « pure » (Blond et al., 2000). 


\section{Patients, matériels et méthodes}

\subsection{Patients et sujets}

L'étude a porté sur 20 patients de sexe masculin porteurs d'un dispositif de neurostimulation à haute fréquence du NST. Les patients étaient suivis dans le service de neurologie du centre hospitalier du pays d'Aix pour l'évaluation postopératoire et le réglage des paramètres de stimulation. Les valeurs des dits paramètres sont rapportées dans le Tableau 1. Au moment de l'étude, les patients étaient en moyenne âgés de $61 \pm 6$ ans, avec une durée de la maladie d'environ $12 \pm 4$ ans ; ils étaient tous en condition sans médicaments antiparkinsoniens au moment des évaluations (n'étant plus sous dopathérapie postopératoire ou bien en sevrage de traitement antiparkinsonien depuis au moins 12 heures). Chaque patient a été enregistré alors dans deux conditions différentes : la neurostimulation arrêtée (condition dite " OFF ») et la neurostimulation en marche (condition dite « ON »). La durée de l'arrêt de la stimulation avant l'évaluation était variable selon le patient, fonction de sa sensibilité propre à l'état OFF ; en moyenne, elle était de 30 minutes.

Onze sujets CTRL, tous de sexe masculin également et avec un âge moyen de $59 \pm 12$ ans, ont aussi participé à l'étude. Tous les patients et sujets CTRL ont été inclus dans l'étude après avoir signé un consentement éclairé.

\section{2. Évaluation aérodynamique}

Nous avons utilisé le système d'évaluation vocale EVA2 (SQLab, Aix-en-Provence) afin de réaliser les enregistrements nécessaires. Des applications logicielles dédiées à l'étude de la dysprosodie ont été conçues pour EVA2, qui fonctionne sous la forme d'une station de travail sur PC dans l'environnement Windows. Le dispositif d'enregistrement comprend un canal acoustique et deux canaux aérodynamiques, ces derniers permettant notamment la mesure de deux paramètres : le débit d'air buccal et la pression intra-orale (PIO). Ainsi, il est possible de mesurer la PIO pendant la tenue d'une occlusive sourde (exemple : «P »). En effet pendant la tenue de l'occlusive sourde, la glotte est ouverte et les PIO et sous glottique s'équilibrent, ce qui permet d'évaluer la PSG via la mesure de la PIO (Fig. 1). Pour ce faire, une sonde d'aspiration est sectionnée pour atteindre une longueur d'environ $3 \mathrm{~cm}$; elle est ensuite connectée au dispositif d'enregistrement et maintenue entre les dents du patient.

Concernant l'enregistrement proprement dit, le patient produisait à débit constant la phrase « Papa ne m'a pas parlé de beau-papa » en ménageant une prise de souffle entre chaque répétition et en veillant à ce que la sonde ne fût ni écrasée par les dents ni obstruée par la salive. Nous étions intéressés par les seconds $\mathrm{P}(\mathrm{P} 2)$, quatrièmes $\mathrm{P}(\mathrm{P} 4)$ et sixièmes $\mathrm{P}(\mathrm{P} 6)$ de la phrase afin de suivre l'évolution de la PIO (donc de la PSG) sur le décours temporel. Le premier P n'a pas été pris en compte car souvent mal prononcé et à peine esquissé par nombre de patients. Parmi l'ensemble des réalisations du patient, le choix de celle sur laquelle les mesures s'effectuaient était guidé par une bonne prise de souffle préalable et une articulation correcte de la réalisation. À partir de là, le logiciel EVA2 effectuait le traitement du signal en fonction des pics de pression générés automatiquement et ajustés manuellement si nécessaire au niveau de P2, P4 et P6. La mesure du débit d'air oral était utilisée pour vérifier si les patients réalisaient une bonne occlusion labiale pendant la tenue des « $\mathrm{P}$ » (Fig. 2).

Tableau 1 - Paramètres de stimulation subthalamique utilisés chez les patients de l'étude. Subthalamic stimulation parameters used for the treatment of the patients involved in the study.

\begin{tabular}{|c|c|c|c|c|c|c|c|c|}
\hline \multirow[t]{2}{*}{ Patients } & \multicolumn{2}{|c|}{ Fréquence (Hz) } & \multicolumn{2}{|c|}{ Amplitude (V) } & \multicolumn{2}{|c|}{$\begin{array}{c}\text { Largeur } \\
\text { d'implusion }(\mu s)\end{array}$} & \multicolumn{2}{|c|}{ Contacts stimulés } \\
\hline & D & G & $\mathrm{D}$ & G & $\mathrm{D}$ & G & D & G \\
\hline 1 & 130 & 130 & 3,2 & 2,5 & 60 & 60 & M 2-3- & M 3- \\
\hline 2 & 130 & 130 & 2,3 & 2,5 & 60 & 60 & M 2- & M 2- \\
\hline 3 & 130 & 130 & 3,3 & 3,3 & 90 & 60 & B $2+3-$ & B $3-2+$ \\
\hline 4 & 185 & 130 & 3,1 & 2,9 & 90 & 60 & M 1- & M 0-1- \\
\hline 5 & 180 & 180 & 3 & 2,4 & 60 & 60 & M 3- & M 2-3- \\
\hline 6 & 130 & 130 & 2,3 & 2,7 & 60 & 60 & M 3- & M 2-3- \\
\hline 7 & 130 & 130 & 2,8 & 3,2 & 60 & 60 & B $1-0+$ & B 1-0+ \\
\hline 8 & 180 & 180 & 2,6 & 3 & 90 & 60 & M 2-3- & M 2-3- \\
\hline 9 & 130 & 130 & 1,4 & 2,6 & 60 & 60 & B 3-2+ & M 2- \\
\hline 10 & 130 & 130 & 2,3 & 2,5 & 60 & 60 & M 1-2- & M 1-2- \\
\hline 11 & 185 & 185 & 3 & 3,6 & 60 & 90 & M 1- & B 3- 2- \\
\hline 12 & 185 & 185 & 3 & 3,5 & 60 & 60 & M 2- & M 2-3- \\
\hline 13 & 185 & 185 & 3 & 3,2 & 60 & 60 & M 1- & M 2- \\
\hline 14 & 130 & 130 & 3,4 & 3,4 & 60 & 60 & M 1- & M 3- \\
\hline 15 & 185 & 185 & 2,2 & 2,5 & 60 & 60 & M 2-3- & M 2-3- \\
\hline 16 & 130 & 130 & 3,2 & 2,7 & 60 & 60 & B $2+1-$ & M 1-2- \\
\hline 17 & 185 & 185 & 3 & 3,2 & 60 & 60 & M 2-3- & M 1- \\
\hline 18 & 130 & 130 & 2 & 3 & 60 & 60 & M 2- & M 1- \\
\hline 19 & 185 & 185 & 3,2 & 3,2 & 60 & 60 & M 1-2- & B $2+3-$ \\
\hline 20 & 130 & 130 & 3,2 & 2,5 & 60 & 60 & M 3- & M 2-3- \\
\hline
\end{tabular}




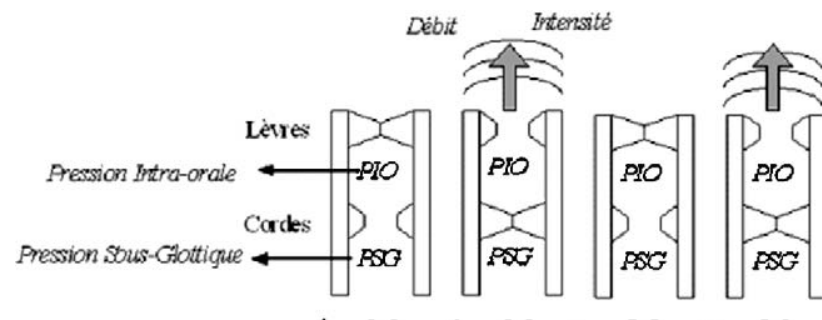

$\begin{array}{llllllll}1 & {[\mathrm{p}]} & 2 & \text { [a] } & 1 & \text { [p] } & 2 & \text { [a] }\end{array}$

Fig. 1 - Évaluation de la pression sous-glottique (PSG). La pression intra-orale (PIO) est équivalente à la (PSG) pendant l'occlusion labiale du phonème " $p$ ". La PSG est estimée de façon indirecte par Airway Interrupted Method (Smitheran et Hixon, 1981), méthode validée notamment par Demolin et al. (1997).

Evaluation of sub-glottal pressure. Intra-oral pressure (IOP) is equivalent to subglottal pressure (SGP) during the labial occlusion of phonem "p" production. Sub-glottal pressure is indirectly estimated by the "Airway Interrupted Method" (Smitheran and Hixon, 1981), a methodology which has been validated notably by Demolin et al. (1997).

\section{3. Évaluation motrice clinique}

Pour chaque patient et avant chaque enregistrement, un neurologue établissait le score moteur de l'Unified Parkinson's Disease Rating Scale (UPDRS ; Fahn et al., 1987) ON et OFF stimulation. Dans cette échelle, l'item 18 correspond à l'évaluation perceptive de la parole et la cotation de la dysarthrie s'étend de zero (parole normale) à quatre (parole inintelligible).

\subsection{Traitements statistiques}

Les comparaisons statistiques entre groupes et entre conditions de stimulation (CTRL vs ON vs OFF) ont été réalisées sur la base d'un modèle linéaire mixte (logiciel « R », version 2.6.2, http://www.r-project.org). Ce modèle est apparu comme le mieux adapté à la configuration de nos comparaisons intergroupes dans le sens où les mêmes patients sont évalués ON et OFF stimulation. Notre objectif, en utilisant ce modèle mixte, était de conserver la différence intrasujet $\mathrm{ON}$ versus OFF en tant qu'indicateur d'effet. De fait, ce modèle a une puissance statistique importante dans notre cas de figure, c'est-à-dire quand la variabilité intersujet est grande, plus large que l'effet estimé. Nous avons défini nos comparaisons statistiques de la manière suivante : les mesures de PIO représentaient le facteur numérique du modèle ; le groupe (CTRL, ON, OFF), la position de la consonne/p/ dans la phrase produite (P2, P4, P6) et le sujet (patients, CTRL) étaient les trois facteurs de variabilité du modèle. Une valeur de $p$ inférieure à 0,05 a été retenue comme seuil de significativité statistique.

\section{Résultats}

\subsection{Scores moteurs de l'Unified Parkinson's Disease Rating Scale}

La moyenne du score UPDRS (partie motrice sur 108) en période ON était de $20,6( \pm 6,4)$ contre $40,9( \pm 12,1)$ en condition

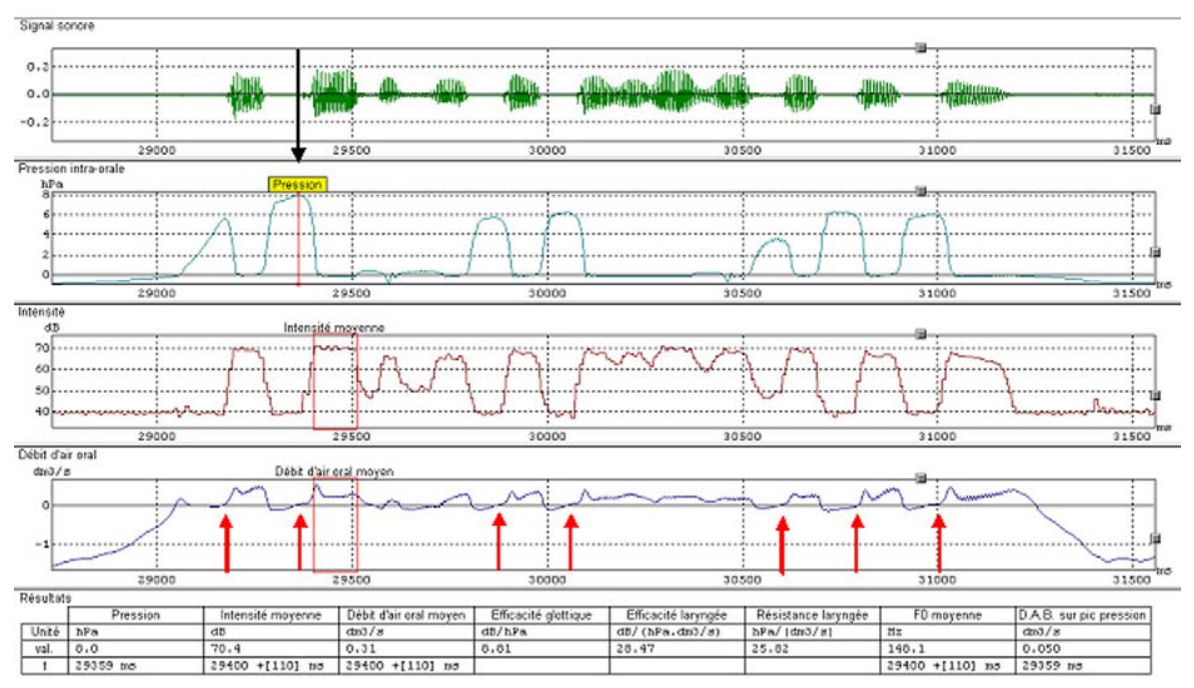

Fig. 2 - Mesures effectuées à partir du système EVA2. De haut en bas : signal audio, PIO, intensité moyenne, débit d'air oral moyen, résultats, fréquence fondamentale moyenne. Concernant la mesure spécifique de la PIO, il suffit de placer le curseur de pression (indiquée par la flèche noire) au bon endroit pour que EVA2 donne immédiatement la valeur correspondante. Au moment de la réalisation des différents « $\mathrm{P}$ » de la phrase, le débit d'air oral moyen est à zéro (flèches rouges), attestant de la bonne occlusion labiale.

EVA2 system measurements. From top/down: audio signal, IOP, mean intensity, mean oral airflow, results, mean fundamental frequency. Regards to the specific IOP measurement, the pressure label (visualised by the black arrow) needs to be placed at the dedicated location in order to give the EVA2 system the opportunity of calculating the corresponding value. At the time of the different "P" realisations in the sentence, the mean oral airflow indicated zero (red arrows), confirming the actual labial occlusion. 


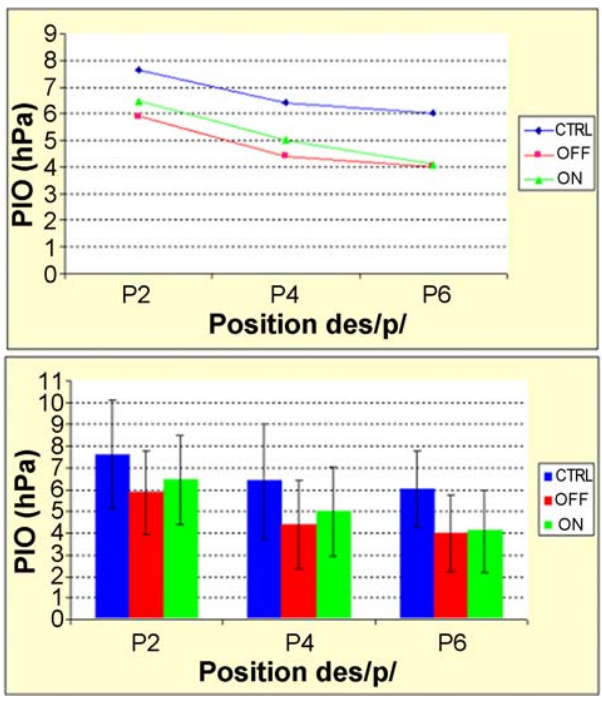

Fig. 3 - Courbes de moyennes des PIO en fonction des points de mesure P2, P4 et P6. En haut : tendance générale chez les sujets contrôles (CTRL), les patients stimulés (ON) et non-stimulés (OFF). En bas : valeurs et écarts types de chaque groupe.

IOP mean tendencies related to P2, P4 and P6 measurement points. Up: general trend in control subjects (CTRL), stimulated (ON) and un-stimulated (OFF) patients. Bottom: values and standard deviations of each group.

OFF. Pour l'item 18 spécifique de la parole, le score en période ON était de $1,3( \pm 0,5)$ contre $1,9( \pm 0,5)$ en OFF.

\subsection{Valeurs de la pression intra-orale intragroupes}

La Fig. 3 donne la tendance de la PIO en fonction des trois points de mesure, aussi bien pour les CTRL que pour les patients en conditions ON et OFF. On constate une tendance générale de la chute de la PIO en allant de P2 à P6 en passant par P4 ; la courbe des CTRL se détache nettement de celles des patients en condition ON et OFF. Il est à noter que les deux courbes ON et OFF sont nettement séparées au niveau des deux premiers points de mesure (P2 et P4) mais ont tendance à converger sur le troisième point (P6). Le Tableau 2 présente les valeurs chiffrées des PIO chez les CTRL et les patients dans les deux conditions OFF et ON, avec également le pourcentage de chute de PIO à partir du premier point de mesure (P2) : ainsi observe-t-on une chute de la PIO de $21,16,31,83$ et $36,61 \%$, respectivement, chez les CTRL, OFF et $\mathrm{ON}$.

\subsection{Comparaisons intra- et intergroupes}

Des différences statistiquement significatives entre les sujets CTRL et les patients ont été observées (Tableau 3). La comparaison ON versus CTRL $(p=0,04)$ se base sur une différence de PIO entre les deux groupes de $1,51 \mathrm{hPa}$; la comparaison ON versus OFF $(p=0,02)$ ne s'appuie que sur une différence de $0,42 \mathrm{hPa}$. Ces écarts de pressions expliquent la morphologie des courbes de tendance de PIO (Fig. 3) déjà observées. En d'autres termes, sur une différence CTRL versus OFF de 1,93 hPa, la stimulation en marche permet de recouvrer $0,42 \mathrm{hPa}$, soit environ $21 \%$ de l'écart séparant la condition OFF des valeurs CTRL. Un effet de position de la consonne « $\mathrm{P}$ » lors de la mesure de PIO a été retrouvé (Tableau 3 ) de façon hautement significative $(p=0,0001)$ : comme précisé précédemment, la PIO chute progressivement de $\mathrm{P} 2$ à $\mathrm{P} 4$ puis à $\mathrm{P} 6$. Le même effet d'ordre est observé quel que soit le groupe considéré (CTRL, OFF ou ON), aucune interaction significative n'ayant été constatée pour les effets combinés groupe et position (Tableau 3).

\section{Discussion}

L'évaluation de la PIO montre qu'elle connaît une baisse significative en condition OFF chez les patients par rapport aux CTRL appariés en âge de manière initiale et persistant tout au long de la production. La stimulation du NST, bien qu'améliorant peu l'item parole de l'UPDRS, entraîne une amélioration significative de la PIO dans la partie initiale de laproduction considérée, donc du cycle expiratoire de la parole.

La MPI, du fait du déficit dopaminergique qui lui est inhérent, induit un dysfonctionnement de la musculature respiratoire responsable en partie de la dysarthrie (Murdoch et al., 1989). En effet il y a, dans le cadre global d'un mauvais contrôle du flux d'air expiratoire, une altération de la quantité d'air nécessaire à la vibration des cordes vocales (Solomon et Hixon, 1993 ; Jiang et al., 1999). Or, la PSG est le résultat d'une poussée de la colonne d'air par la pression pulmonaire, avec une résistance laryngée (Crevier-Buchman, 2007 ; Solomon, 2007). Dans le cadre particulier de cette étude, quand on mesure la PSG via la PIO, la glotte est ouverte ; à ce moment donc c'est un gradient de pression qui est mesuré et non une valeur statique. Ce gradient est le résultat d'une action coordonnée entre les muscles respiratoires et l'étage laryngé, témoin de la coordination pneumophonique. Dans la MPI, la chute de la pression pulmonaire associée aux mouvements hypokinétiques des muscles laryngés induit une altération de la PSG : nous avons donc montré dans cette étude qu'il est

Tableau 2 - Tendance de la décroissance des courbes de mesures de PIO pour les groupes ON, OFF et CTRL. Tendencies for decrease of IOP measurements for ON, OFF and CTRL groups.

\begin{tabular}{lccccc} 
& P2 $(\mathrm{hPa})$ & $\mathrm{P} 4(\mathrm{hPa})$ & $\mathrm{P} 6(\mathrm{hPa})$ & $\begin{array}{c}\text { Différence } \\
(\mathrm{P} 2-\mathrm{P} 6)(\mathrm{hPa})\end{array}$ & $\begin{array}{c}\text { Pourcentage de décroissance } \\
([\mathrm{P} 2-\mathrm{P} 6] / \mathrm{P} 2) * 100\end{array}$ \\
\hline CTRL & & & 6,03 & 1,62 & $-21,16$ \\
Stimulation OFF & 7,64 & 6,40 & 4,00 & 1,87 & $-31,83$ \\
Stimulation ON & 5,87 & 4,38 & 4,08 & 2,36 & $-36,61$ \\
\hline
\end{tabular}


Tableau 3 - Comparaisons statistiques des mesures de PIO en fonction des groupes d'étude (OFF, ON et CTRL) et des points de mesures (P2, P4, P6).

Statistical comparisons of IOP measurements related to the different study groups (OFF, ON and CTRL) and measurement points (P2, P4, P6).

\begin{tabular}{lcc} 
Comparaison & $\begin{array}{c}\text { Valeurs } \\
\text { estimées des } \\
\text { écarts de } \\
\text { pression (hPa) }\end{array}$ & $\begin{array}{c}\text { Valeurs } \\
\text { de } p\end{array}$ \\
\hline CTRL-ON & 1,51 & 0,04 \\
OFF-ON & $-0,42$ & 0,02 \\
Effet de position P2, P4, P6 & $-1,66$ & 0,0001 \\
Interaction (CTRL vs ON) vs position & 0,52 & 0,17 \\
Interaction (OFF vs ON) vs position & 0,35 & 0,27 \\
\hline
\end{tabular}

possible de considérer la PSG, ou encore la PIO, comme un indice fort de la dysarthrie parkinsonienne en général, et de son versant pneumophonique en particulier.

La chute de la PIO entre P2 et P6 est moins importante pour les sujets CTRL que pour les patients, attestant d'une meilleure maîtrise de la coordination pneumophonique. Pour les patients, paradoxalement, la chute de la PIO entre P2 et P6 est plus importante en condition ON qu'en condition OFF : cela pourrait s'expliquer par une sorte d'« effet plancher » dans la mesure où les patients en condition OFF partent de niveau de PIO plus bas à l'origine ; leur marge de décroissance est alors nécessairement plus étroite, le niveau final " plancher » étant sensiblement le même pour les deux conditions ON et OFF.

La stimulation du NST, comme en attestent les scores UPDRS en conditions ON et OFF STIM, améliore l'état moteur des patients de l'ordre de $54 \%$, ce qui n'est pas loin des $60 \%$ de Blond et al. (2000). Ce résultat est conforme aux nombreux résultats dans la littérature qui mentionnent l'impact favorable de la stimulation du NST sur les signes moteurs de la maladie (Limousin et al., 1998 ; Pollak et al., 1993). De la même façon, d'autres travaux ont conclu à l'effet réellement bénéfique de la stimulation du NST sur les troubles axiaux (Gentil et al., 1999 ; Ciucci et al., 2008 ; Yamada et al., 2008). Toutefois, dans la présente étude, la PIO est restaurée seulement sur la partie initiale de la phrase prononcée par les patients ON par rapport à la condition OFF. Cette restauration de PIO en ON, au-delà de son caractère partiel, est très en deçà de celle des sujets CTRL (Fig. 3 et Tableau 3) ; cela suggère que l'effet global de la stimulation du NST sur les troubles de la parole reste médiocre et souvent ambigu. En effet, même pour les études qui ont montré un impact nettement positif de la stimulation du NST sur la parole (Gentil et al., 1999 ; Gentil et al., 2001), il n'existait pas toujours une homogénéité quant à la différence significative, statistiquement parlant, de certains paramètres (intensité, durée et précision de contraction musculaire stabilisée) entre les deux conditions $\mathrm{ON}$ et OFF. Nos résultats vont dans le même sens avec des valeurs de PIO en moyenne globalement supérieures pour la condition ON par rapport à la condition OFF au niveau des deux premiers points de mesure (P2 et P4) et un effet de convergence des courbes vers le troisième point (P6) (Fig. 3). L'effet de la stimulation sur la PIO est donc limité et transitoire, reposant le problème plus général des mécanismes d'action encore mal connus de la stimulation du NST sur les signes de la MPI, à savoir la frappante dichotomie entre l'atténuation franche de la triade motrice classique (akinésie, rigidité, tremblement) et l'amélioration plus discrète, voire l'aggravation, des signes axiaux tels que la dysarthrie. Des variables comme la localisation des électrodes au sein du NST et les paramètres de stimulation pourraient représenter une tentative d'explication (Pinto et al., 2005 ; Törnqvist et al., 2005) mais aucune certitude réelle n'existe à ce jour sur le pourquoi de cette dichotomie. À énergie totale délivrée équivalente (Koss et al., 2004), un travail récent a mis en évidence une efficacité supérieure de la stimulation du NST sur certains signes axiaux, notamment le freezing au cours de la marche, avec des fréquences à $60 \mathrm{~Hz}$ en comparaison avec des fréquences à $130 \mathrm{~Hz}$ (Moreau et al., 2008). Notre groupe de patients ayant été stimulé entre 130 et $185 \mathrm{~Hz}$ ne permet pas d'étudier l'effet de la variation de fréquence de stimulation sur les paramètres considérés : la recherche des fréquences "idéales " de stimulation pour une meilleure restauration des troubles de la parole devrait faire l'objet d'un travail ultérieur.

Dans un travail de Nambu et al. (1996) sur le singe macaque, à partir de techniques de microstimulation et d'injections de traceurs antérogrades, il a été mis en évidence une double représentation somatotopique au sein du NST : la partie latérale du NST a des connexions avec le cortex moteur primaire alors que la partie médiale du même noyau est plutôt en rapport fonctionnel avec l'aire motrice supplémentaire (AMS). Si le NST exerce une action sur le cortex via le complexe globus pallidus interne/substantia nigra pars reticulata (GPi/SNr) d'abord et ensuite le thalamus, l'existence de ces connexions directes entre NST et cortex moteur primaire, d'une part, et entre NST et AMS, d'autre part, pourrait permettre d'envisager une modulation directe de l'activité neuronale corticale par la stimulation du NST. Dans une telle hypothèse, on pourrait concevoir que la face et les membres, mieux représentés topographiquement selon l'homonculus de Penfield au niveau cortical et au niveau du NST (Rodriguez-Oroz et al., 2001), soient modulés plus facilement que le tronc plus faiblement représenté topographiquement ; cette divergence somatotopique pourrait être une explication de la dichotomie résultante de l'action de la stimulation du NST sur les signes moteurs de la MPI. La modulation de l'activité neuronale corticale par la stimulation du NST pourrait relever d'un index de représentation topographique où les parties du corps les plus largement représentées au niveau du cortex moteur primaire seraient les plus sensibles à la stimulation. Ce modèle pourrait ainsi expliquer l'amélioration partielle des signes axiaux de la MPI par la stimulation du NST.

\section{Conclusion}

Il apparaît important de considérer la mesure de PIO comme un indicateur pertinent de la dysarthrie parkinsonienne. Ce paramètre, témoin de la coordination pneumophonique, n'est sujet qu'à une amélioration limitée et transitoire par la stimulation du NST : cela confirme à nouveau l'effet controversé de la stimulation du NST sur les troubles axiaux et prouve la nécessité d'investigations plus approfondies afin de mieux comprendre les mécanismes d'action de cette technique sur les troubles en question. 


\section{Conflits d'intérêts}

Aucun.

\section{Remerciements}

Ce travail n'aurait pas été possible sans l'obtention d'un Programme hospitalier de recherche clinique (PHRC) régional (2003) et le soutien de l'Association France Parkinson : qu'ils veuillent trouver ici nos sincères remerciements.

Les auteurs souhaitent aussi remercier Robert Espesser (LPL, Aix-en-Provence) pour son aide précieuse dans l'analyse statistique des résultats.

\section{RÉ FÉ REN C ES}

Blond S, Touzet G, Krystkowiak P, Defebvre L, Destee A. Le traitement neurochirurgical de la maladie de Parkinson : quelle technique retenir ? Rev Neurol (Paris) 2000;156 [S2b257-]

Ciucci MR, Barkmeier-Kraemer JM, Sherman SJ. Subthalamic nucleus deep brain stimulation improves deglutition in Parkinson's disease. Mov Disord 2008;23:676-83.

Crevier-Buchman L. Modélisation du fonctionnement laryngé. In: Auzou P, Monnoury-Rolland V, Pinto S, Özsancak C, editors. Les dysarthries. Marseille: Solal; 2007. p. 91-100.

Darley FL, Aronson AE, Brown JR. Differential diagnostic patterns of dysarthria. J Speech Hear Res 1969;12:246-69.

Demolin, D., Giovanni A, Hassid, S., Heim, C,, Lecuit, V., Socquet A. 1997. "Direct and indirect measurements of subglottic pressure", Proc. Larynx. 97, Marseille, pp. 69-72.

Fahn S, Elton RL, committee motUd. Unified Parkinson's disease rating scale. In: Fahn S, Marsden CD, Calne DB, editors. Recent developments in Parkinson's disease. Florham Park, NJ: MacMillan, Health Care Information; 1987. p. 153-64.

Gentil M, Garcia-Ruiz P, Pollak P, Benabid AL. Effect of stimulation of the subthalamic nucleus on oral control of patients with parkinsonism. J Neurol Neurosurg Psychiatry 1999;67:329-33.

Gentil M, Chauvin P, Pinto S, Pollak P, Benabid AL. Effect of bilateral stimulation of the subthalamic nucleus on Parkinsonian voice. Brain Lang 2001;78:233-40.

Jiang J, O'Mara T, Chen HJ, Stern JI, Vlagos D, Hanson D. Aerodynamic measurements of patients with Parkinson's desease. J Voice 1999;13:583-91.

Limousin P, Krack P, Pollak P, Benazzouz A, Ardouin C, Hoffman $D$, et al. Electrical stimulation of the subthalamus nucleus in advanced Parkinson's disease. N Engl J Med 1998;339:1105-11.

Koss AM, Alterman RL, Tagliati M, Shils JL. Calculating total electrical energy delivered by deep brain stimulation systems. Ann Neurol 2004;56:290-4.
Moreau C, Defebvre L, Destée A, Bleuse S, Clement F, Blatt JL, et al. STN-DBS frequency effects on freezing of gait in advanced Parkinson disease. Neurology 2008;28: 80-4.

Murdoch BE, Chenery HJ, Bowler S, Ingram JC. Respiratory function in Parkinson's subjects exhibiting a perceptible speech deficit: a kinematic and spirometric analysis. J Speech Hear Disord 1989;54:610-26.

Nambu A, Takada M, Inase M, Tokuno H. Dual somatotopical representations in the primate subthalamic nucleus: evidence for ordered but reversed body-map, transformationsfrom the primary motor cortex and the supplementary motor area. J Neurosci 1996;16: 2671-83.

Pollak P, Benabid AL, Gross C, Gao DM, Laurent A, Benazzouz A, et al. Effets de la stimulation du noyau sous-thalamique dans la maladie de Parkinson. Rev Neurol (Paris) 1993;149:175-6.

Pinto S, Gentil M, Krack P, Sauleau P, Fraix V, Benabid AL, et al. Changes induced by levodopa and subthalamic nucleus stimulation on Parkinsonian speech. Mov Disord 2005;20:1507-15.

Rodriguez-Oroz MC, Rodriguez M, Guridi J, Mewes K, Chockkman V, Vitek J, et al. The subthalamic nucleus in Parkinson's disease: somatotopic organization and physiological characteristics. Brain 2001;124: 1777-90.

Selby G. Parkinson's disease. In: Vinken PJ, Bruyn GW, editors. Diseases of the basal ganglia Handbook of clinical neurology, 6. Amsterdam: North Holland Publ Co; 1968.

Smitheran J, Hixon T. A Clinical method for estimating laryngeal airway resistance during vowel production. J Speech Hear Disord 1981;46:138-46.

Solomon NP, Hixon TJ. Speech breathing in Parkinson's disease. J Speech Hear Res 1993;36:294-310.

Solomon NP. La fonction respiratoire dans la production de parole. In: In Auzou P, Monnoury-Rolland V, Pinto S, Özsancak C, editors. Les dysarthries. Marseille: Solal; 2007. p. 44-55.

Törnqvist AL, Schalén L, Rehncrona S. Effects of different electrical parameter settings on the intelligibility of speech in patients with Parkinson's disease treated with subthalamic deep brain stimulation. Mov Disord 2005;20:416-23.

Vercueil L. Les critères de diagnostic clinique de la maladie de Parkinson et des syndromes parkinsoniens : revue de la littérature. Rev Neurol (Paris) 2000;156:S2b14-22.

Viallet F, Gayraud D, Bonnefoi-Kyriacou B, Dupel-Pottier C, Aurenty R. Aspects cliniques et thérapeutiques de la maladie de Parkinson. Encycl Med Chir/Neurology 2001;26 [17-060-A-50].

Viallet F, Teston B. La dysarthrie dans la maladie de Parkinson. In: Auzou P, Monnoury-Rolland V, Pinto S, Özsancak C, editors. Les dysarthries. Marseille: Solal; 2007. p. 375-82.

Yamada K, Goto S, Hamasaki T, Kuratsu JI. Effect of bilateral subthalamic nucleus stimulation on levodopaunresponsive axial symptoms in Parkinson's disease. Acta Neurochir (Wien) 2008;150:15-22. 